$\xi=1$ 圆

\title{
A Lightweight One Time Password (OTP) Based Smart Learning in Internet of Things
}

\author{
Nithin Rao Kadham ${ }^{1 *}$, Sreenivasa Ravi.K ${ }^{2}$ \\ ${ }^{1}$ Research Scholar, KL E F (Deemed to be University), VADDESWARAM, GUNTUR, A.P. University, India \\ ${ }^{2}$ Professor, KL E F (Deemed to be University), VADDESWARAM, GUNTUR, A.P. University, India \\ *Corresponding authorE-mail: nithin@learningsteps.in, ravi.kavuluri@kluniversity.in
}

\begin{abstract}
The Internet of Things is another worldview that is changing processing. It is expected that all items around us are associated with the system, giving "whenever, anyplace" access to data. This idea is making progress, because of advances in nanotechnology which permits the making of gadgets fit for interfacing with the Internet productively. Presently a day countless are associated with the web, running from cell phones to machines. In this paper we concentrate on the instruction field, this paper manages the use of the ideas of Internet of Things and its application in making brilliant condition. The particular objective is to plan a keen situation for upgrading the instructing and learning forms at colleges. The earth ought to incorporate sufficient ideas of keen structures and brilliant classrooms with e-learning frameworks, to furnish understudies with cutting edge e-learning administrations and administrations that enhance the general nature of understudies' understanding. In this paper, we have proposed a safe biometric, One Time Password (OTP) and ace key based validation framework in IoT organize. The confirmation convention utilizes XOR operation and lightweight hash operation. The security examination demonstrates that it is safe against various assaults.
\end{abstract}

Keywords: Accumulated Hashing; e-learning; Internet of Things (IOT); One Time Password (OTP); Secure Authentication.

\section{Introduction}

Internet of Things empowers interconnecting shrewd gadgets. For example, controllers, sensors with other data correspondence foundation [1][2]. Utilizing of this savvy gadgets can be guides the expand level of the computerizing assignments, this cause increasing the better profitability in wide range of conditions. Savvy conditions, for example, keen homes, shrewd classrooms or brilliant manufacturing plants, are framed by associating and including countless gadgets to a current correspondence framework. In instruction circle, IoT has wide range of application, which can not sufficiently utilize. IoT advancements are necessary piece of the shrewd learning condition, for example, keen classrooms. Savvy classroom is an idea which coordinates a few data and correspondence innovations to empower synergistic learning so as to enhance the general learning and showing forms [3].

Diverse innovations can be utilized for conveying a shrewd classroom, for example, NFC, brilliant cell phones, interactive media gadgets and so forth. Moreover, learning condition ought to be charming spot for educating and learning. Hence, brilliant classroom ought to be furnished with frameworks for cooling, lighting, and warming and nearness administration.

The hardware equipment in shrewd classrooms are normally overseen by sufficient programming. Presence of a stage that incorporates all administrations in extent of keen learning condition is vital for the instructors and understudies.

To get to these administrations, numerous applications are created and human are utilizing those applications in their brilliant gadgets. Additionally numerous exercises are controlled by savvy gadgets, for example, Lockitron can bolt and open entryways by cell phone
[2], medicinal services data can be checked by advanced mobile phone.

Because of smooth administration, PDAs are putting away delicate individual data to its memory. That is the reason this field is ending up more inclined to assault. There may probability of security or data spillage, fraud, neglecting to ensure privacy, honesty, genuineness and so on.

We know we can actualize verification benefit utilizing both of three variables: something definitely known to us (e.g., secret word), having something (e.g., shrewd card), something we are (biometric data, e.g., unique mark, iris examine and so on). Customary confirmation framework utilizes single factor implies what we know (e.g., password).

Besides these elements, One Time Pad (OTP) is likewise utilized as a moment factor to validate the clients. To guarantee security in validation we can apply barrier inside and out idea by including various elements like biometric data alongside One Time Password (OTP). In this paper, we have proposed OTP based secure verification and key assertion convention to guarantee the safe access amongst clients and IoT hubs.

The IOT has its own specific challenges, which ought to be tended to. Every contraption will require an IP convey to pass on, the present IPv4 has only 4.3 billion exceptional areas, which will be exhausted soon and thusly we should conform to IPv6. The accompanying test would be data amassing; as billions of contraptions are interfacing the data ought to be secured for which tremendous storage space is required. After the data have been assembled we need to guarantee that the security approaches are set up as more individual information will be accumulated from contraptions which not get cracked and the data should not get in the 
hands of software engineers. Insurance would moreover be an unprecedented test as after the present hacks people are ending up more stressed over their security. In this manner these troubles ought to be taken in careful idea before orchestrating any wander related to the loT. In this endeavor of development distinguishing proof these security challenges have been considered [2].

This exploration speaks to an improvement of a stage for brilliant learning condition. These IoT stage gathers information from the keen learning condition from different controllers, sensors and microcomputers. The point of this exploration is improving learning of Internet of things in a scholastic domain by making ventures in created IoT stage.

The university of Belgrade, at the faculty of science, examine the extent of E-business (E-LAB).. The IOT ELAB stage was produced to enable understands; learn of IoT to accomplish improved learning results.

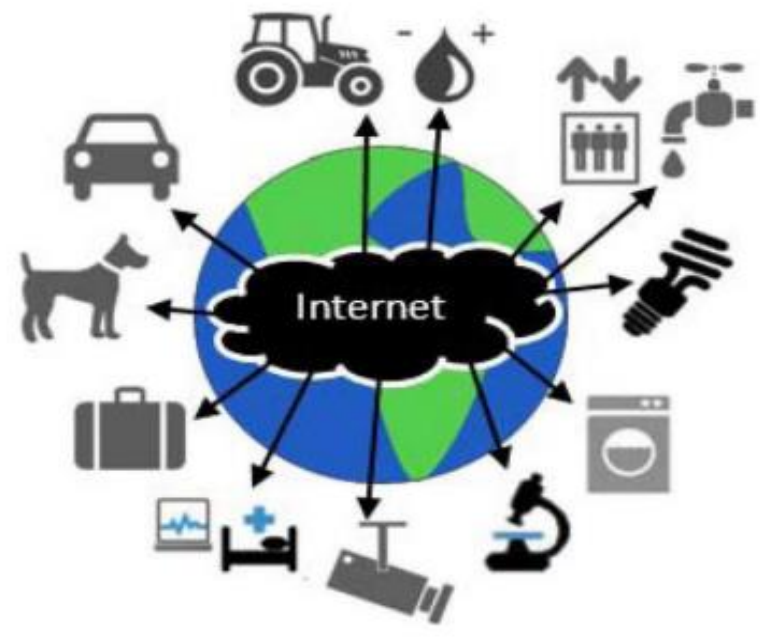

Fig 1.1: IOT used in different fields

\subsection{Objective}

Indeed, guaranteeing security of information trade is among the considerable difficulties of the Internet of things. Here we try to present another grouping of assaults in consistence with the OSI layers and the goal of security(OTP) that we look to accomplished so as to create novel methods and procedures to battle against these assaults.

The primary target of keen situations as a piece of more extensive term, Internet of Things, is to make regular daily existence simpler.

$>$ From the sensor, analyzing and processing the historical data

$>$ Devices can receive notifications and alerts (OTP)

\subsection{Problem Statement}

In this concept, we are managing the security applications. Security in validation is principal concern. We have completed a casual security investigation of the proposed convention. This convention demonstrates that it is safe against various assaults like stolen gadget assault, replay assault, imitates assault and so on. It additionally gives common validation and client secrecy

\section{Literature Review}

IOT can be characterized as an inexactly decentralized framework, coupled, made from savvy objects /self-governing physical or advanced system prepared articles which can gather natural information and to process these information [4]. The Internet turns into a system of all gadgets, not exclusively PCs.
From the Gartner's forecasts, almost 26 billion of gadgets connected things in the year 2020 [5].

The term "Web of Things" is normally utilized for gathering controllers, sensors and microcomputers into shrewd situations.

Sensors are simple and advanced gadgets, recognize physical normal for nature, for example, temperature, stickiness, weight, levels of sound noise and so forth [6]. Actuators are works like switches - which can control different gadgets. Sensors and actuators are insufficient without anyone else for making keen situations. They are regularly utilized together with more perplexing gadgets. In the circle of the IoT, a microcontroller stage is Arduino and microcomputer is Raspberry Pi.

An essential part of the IoT is interfacing with different systems. In the time of broadband advances, for example, Wi-Fi and LTE, these issues are particularly developing. Information gave by various gadgets ought to be accessible each time and all over the place. By making a satisfactory stage in the cloud, it is conceivable to coordinate various information sources.

The realty of the Internet of Things can be seen from two viewpoints: the Internet perspective, which concentrates on giving sufficient Internet administrations, and the things angle, which incorporates gathering and handling information gained from gadgets in view of legitimate OTP. Keen gadgets will be enter components in programming created by utilizing the protest arranged engineering.

The IoT stage can associate a sensor foundations which speak to information generators with customers intriguing in acquiring information which speak to buyers. Sensor framework can contain one or numerous sensors. They can be versatile and associated with a similar cloud remotely. Information procured by utilizing the sensor framework are put away into a non-social database. Customers are capable at that point to get to these information [7]. Database can be delocalized and conveyed keeping in mind the end goal to trade and store data.

These days, IoT stages depend on cloud foundation. For the most part these stages are utilized for gathering information from sensors and other shrewd gadgets from nature in which they are executed. Cloud administrations and assets can be conveyed by three cloud benefit models [8][9][10]: Platform as a Service (PaaS), Software as a Service (SaaS) and Infrastructure as a Service (IaaS) The IoT PaaS. Platform as a Service empowers the engineers to devour the assets in IaaS and convey their applications onto a virtualized cloud stage [9]. The standout among the most broadly utilized PaaS is a Xively. This is a free online administration empowering engineers, to send their own particular application in view of the IoT and information procured from sensors.

\section{IOT and Security Features}

This vision of the IoT will acquaint another measurement with the data and correspondence advances. Notwithstanding the two worldly and spatial measurements that enable individuals to interface from anyplace whenever, we will have another "protest" measurement that will enable them to associate with anything. The IoT will cover an extensive variety of utilizations and nearly touch all regions that we confront each day. This will permit the rise of brilliant spaces around a universal figuring. These keen spaces include: urban areas, vitality, transport, wellbeing, industry, and farming, and so on. (Mitchell et al., 2013). 

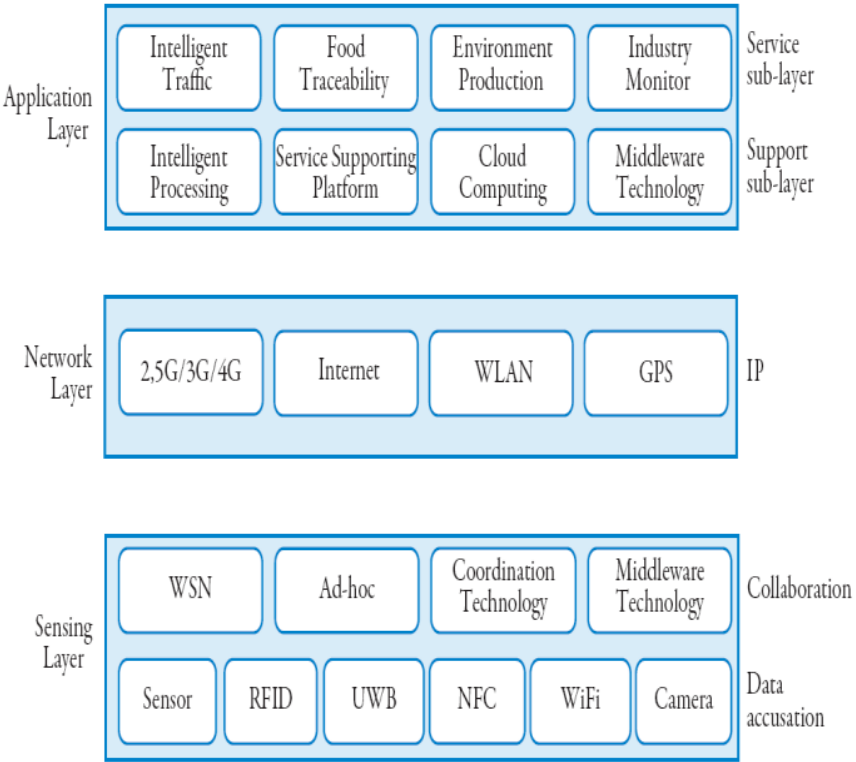

Figure 2: The IoT architecture model

The IoT is portrayed by a far reaching discernment, a dependable transmission and astute preparing. Figure. 2 demonstrates the three-layer design of IoT : applications, system and detecting layer. The detecting understands a far reaching discernment by gathering ongoing dynamic information through different sensors (counting labels) while the system layer is principally in charge of the dependable information transmission, transferring information gained from the detecting layer to the application layer. Utilizing conveyed registering advancements, including distributed computing, the application layer performs gigantic information handling and shrewd investigation with the end goal of wise control (Zheng et al., 2011).

\section{Security:}

As Wireless systems wind up noticeably omnipresent and their security turns into a vital outline of a protected arrangement that should meet some fundamental and critical prerequisites. We fundamentally concentrate on security necessities, and after that we address the principle security issues so as to guarantee the arrangement of a protected IoT.

The essential arrangement of security administrations including:

$>$ Authentication: The way toward deciding if somebody or something is, indeed, who or what it is announced to be. We recognize two sort of assaults identified with validation to be specific, pantomime assault where an aggressor puts on a show to be another substance, and Sybil assault where the assailant utilizes diverse personalities in the meantime.

$>$ Authorization: The way toward giving somebody authorization to do or have something.

> Integrity: Set of means and systems to limit the adjustment of information to approved people. Assaults identified with information uprightness are message change assault and message creation assault.

$>$ Confidentiality: Concept to guarantee that data must be perused by approved people. Assaults on secrecy comprise of getting to wrongfully to classified information.

$>$ Non-revocation: Set of means and methods to demonstrate the contribution of a substance in an information trade. Assaults on non-disavowal comprise of a foreswearing of cooperation in all or part of correspondences.

$>$ Availability: the goal is to ensure the survivability of system administrations against Denial-of-Service as- saults. The assault going for an aggregator can make some piece of the system misfortunes its accessibility in light of the fact that the aggregator is capable to give the estimation of that system part.

$>$ Privacy: The target of this security prerequisite is to keep private data from being spilled to malevolent elements. Assaults on security are identified with illicitly assembling delicate data about elements (e.g., listening stealthily).

\section{Implementation}

\subsection{OTP Authentication}

With the expansion of client verification benefit and that of assaults to the conventional confirmation technique, the requirement for enhanced security of client validation strategy develops. Conventional static secret key verification strategies are broadly utilized because of their accommodation. Be that as it may, they regularly experience the ill effects of assaults as listening in, replay, speculating et cetera. As an approach to free from any of them, OTP confirmation benefit is normally embraced to help 2factor validation for different fields, for example, money related, gateway, diversion et cetera $[4,5]$.

One-time watchword validation, as a matter of course, requires a client side OTP generator, called token, for the age of dynamic passwords. As of late, the OTP age in cell phones is as a rule progressively utilized as an approach to encourage OTP verification without devoted OTP equipment token. This is alluded to as portable OTP token. Be that as it may, the versatile OTP as a rule is an application actualized by programming in a portable terminal. That is the reason there is a plausibility in which vital data or the OTP esteem is hacked by an outer assault for producing a versatile OTP.

\subsection{OTP Generation \&Implementation Application}

To create OTP esteem in light of the extended IOT with the OTP age motor, the OTP age application on the gadget is composed as appeared in Fig. 3. There are four primary useful pieces: IOTbased OTP age motor, OTP age API (Application Programming Interface), OTP administration API, and UI.

In the OTP age process the application on the gadget just assumes the part of demonstrating the OTP age ask for and the created OTP incentive to the client. Practically speaking, the production of the OTP esteem and the capacity of the essential information are performed on the free equipment IOT. Therefore, it is conceivable to create OTP safely.

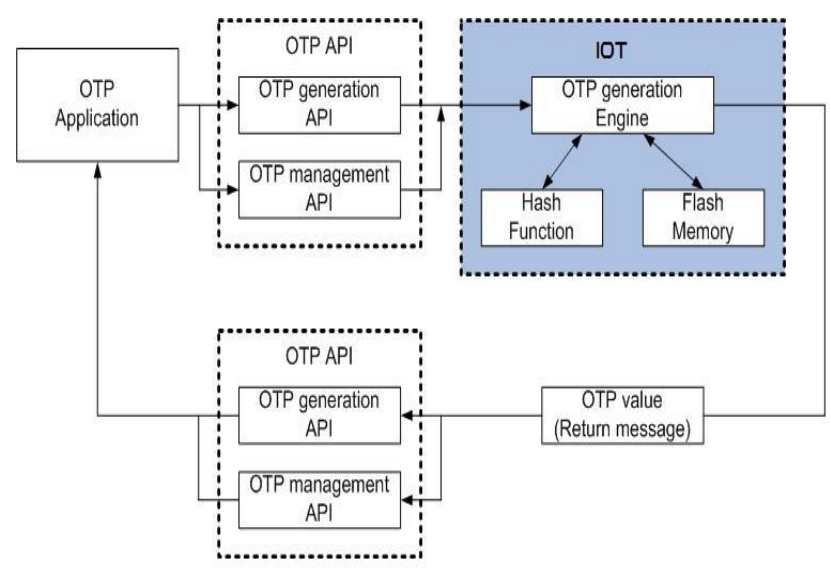

Fig 3: OTP application 


\subsection{Verification}

To confirm the IOT-based OTP age work, a reference OTP application and an OTP confirmation server accommodated OTP check are used [9]. Since the reference application is actualized by programming just, it is adjusted by the outline of the equipment based OTP age so that the OTP age work and the critical information stockpiling capacity can be performed in conjunction with the OTP age motor of the IOT [11-14].

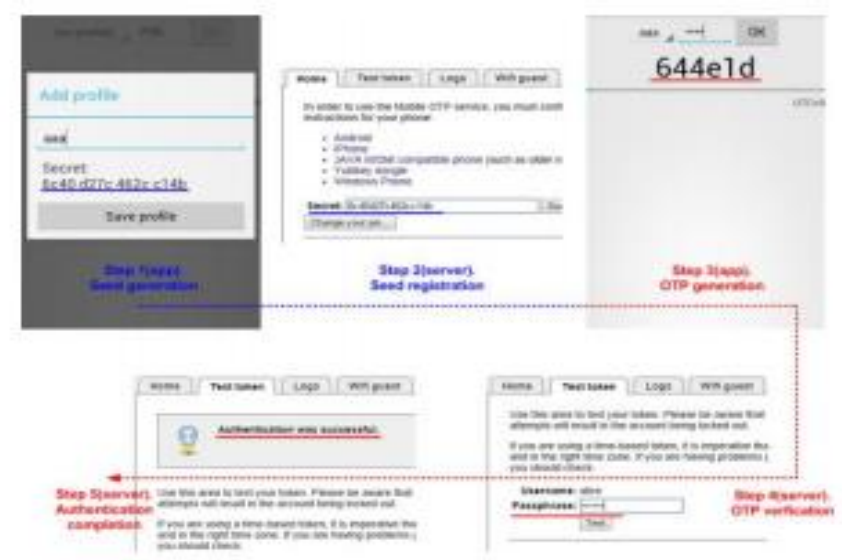

Fig. 4: Verification procedure of OTP generation

Fig.4 shows the confirmation strategy in which the created OTP esteem from the altered OTP age application on the model gadget with IOT is checked at the verification server.

\section{Conclusion}

This paper shows the plan and execution of an effective OTP age motor for IoT customer gadgets that requires both the security of gadgets and that of administrations. For the reason, each of the IOT and the OTP age application is adjusted keeping in mind the end goal to include a novel equipment based OTP motor to the IoT and to outline the OTP age application interworking with the extended IoT. We additionally display another strategy to execute the equipment/programming co-outline of OTP age in IoT gadgets. The consequences of execution and check demonstrate that the proposed design contributes a decent answer for pragmatic usage of the OTP validation for IoT security.

This arrangement gives an assurance against \replay assaults", in light of the fact that the traded OTPs depend on irregular numbers, in this manner, they are substantial just for one exchange. Utilizing the boycotting instrument we can secure our frameworks against \some DoS" assaults. At long last it is flexible and doesn't diminish adaptability of the framework. It can be sent in di_erent WSNs advancements, while keeping a similar level of strength.

\section{References}

[1] ITU.: The Internet of Things. Internet Reports, (2005).

[2] J. Guaus, L. Kanniainen, P. Koistinen, P. Laaksonen, K. Murphy, J. Remes, N. Taylor and O. Welin, "Best Practice for Mobile Financial Services: Enrolment Business Model Analysis. Mobey Forum Mobile Financial Services Ltd., Helsinki, (2008).

[3] M. Kim, H. Ju, Y. Kim, J. Park and Y. Park, "Design and implementation of mobile trusted module for trusted mobile computing", IEEE Transactions on Consumer Electronics, vol. 56, no. 8, (2010), pp. 134-140.

[4] N. Haller, C. Metz, P. Nesser and M. Straw, "A One-Time Password system", IETF RFC 2289, (1998).

[5] ITU-T.: Management framework of a one time password-based authentication service. Recommendation X.1153, (2011).

[6] R. H. Weber, "Internet of things - new security and privacy challenges", Computer Law \& Security Review, vol. 26, (2010), pp. 23-30.
[7] D. Gessner, A. Olivereau, A. S. Segura and A. Serbanati, A. Trustworthy Infrastructure Services for a Secure and Privacyrespecting Internet of Things. In: Proceedings of IEEE 11th International Conference on Trust, Security and Privacy in Computing and Communications, pp. 998--1003 (2012)

[8] Keoh, S., Kumar, S., Tschofenig, H.: Securing the internet of things: A standardization perspective. In: IEEE Internet of Things Journal, Vol. 1, No. 3, pp. 265--275 (2014)

[9] Mobile OTP project, http://motp.sourceforge.net/

[10] Design and Implementation of Secure OTP Generation for IoT Devices ,Young-Sae Kim1 and Jeong-Nyeo Kim1,2017.

[11] Vudatha, C.P., Nalliboena, S., Jammalamadaka, S.K.R., Duvvuri, B.K.K., Reddy, L.S.S., Automated generation of test cases from output domain of an embedded system using Genetic algorithms, ICECT 2011 - 2011 3rd International Conference on Electronics Computer Technology 5,5941989, pp. 216-220

[12] Sastry, J.K.R., Ganesh, J.V., Bhanu, J.S., I2C based networking for implementing heterogeneous microcontroller based distributed embedded systems, Indian Journal of Science and Technology, Volume 8, Issue 15, 2015

[13] Sastry, J.K.R., Naga Sai Tejasvi, T., Aparna, J., Dynamic scheduling of message flow within a distributed embedded system connected through a RS485 network, ARPN Journal of Engineering and Applied Sciences, Volume 12, Issue 9, 1 May 2017, Pages 2809-2817

[14] Sastry, J.K.R., Suresh, A., Bhanu, S.J., Building heterogeneous distributed embedded systems through rs 485 communication protocol, ARPN Journal of Engineering and Applied Sciences, 2015, 10(16), pp. 6793-6803 